\title{
An Innovative Control Allocation Scheme to Address Reaction Thruster Interactions on a $3 \mathrm{U}$ CubeSat
}

\author{
Jing Pei* \\ NASA Langley Research Center, Hampton, Virginia, 23681 \\ Marc Choueri ${ }^{\dagger}$ Ryan Elandt ${ }^{\ddagger}$ Mason Peck ${ }^{\S}$ \\ Mechanical and Aerospace Engineering Department, Cornell University, Ithaca, New York, 14853 \\ Peter Finch \\ Mechanical and Aerospace Engineering Department, New York University, New York, NY, 10003
}

\begin{abstract}
A wealth of literature exists on control allocation algorithms for over-actuated air vehicles, launch vehicles, and spacecrafts. Most of these algorithms focus primarily on minimizing some objective function such as command tracking error and/or control effector usage. Linear allocators (pseudo inverses) are usually the conventional choice due to their simplicity and the ability to achieve a significant portion of the theoretical moment/impulse space. Generally, it is assumed that there exists minimal interaction effects between control effectors. In fact, very few studies address the problem of control effector interactions in the context of control allocation, especially for small spacecrafts with a reaction control system (RCS). This paper presents a CubeSat RCS design with a four thruster tetrahedral layout such that when two or more thrusters fire, the resultant impulse differs noticeably compared to the sum of the contributions from individual thruster firings. This undesirable effect is caused by the design of the propellant tank and regulator. To mitigate this issue, an innovative modified pseudo inverse (MPI) control allocation algorithm was developed that adjusts the pseudo inverse solution based on test data. The algorithm is iteration-free and superior to the standard pseudo inverse in minimizing the command tracking error.
\end{abstract}

\section{Introduction}

The assembly of multiple small scale spacecrafts in space into large structures such as a space telescope or solar panels could be tremendously beneficial for the science and engineering communities. The Onorbit Autonomous Assembly of Nano-satellites (OAAN) team ${ }^{1-3}$ at NASA Langley Research Center aims to demonstrate autonomous rendezvous and docking of two $3 \mathrm{U}$ CubeSats. The philosophy of the OAAN mission is to use the minimal suite of sensors and actuators while still meeting the mission objectives. For orbital control, the four thruster RCS layout shown in Fig. 1, is intended to provide pure translational motions. The thrusters are strategically placed at the corners of an imaginary tetrahedron (middle $\mathrm{U}$ of the $3 \mathrm{U}$ CubeSat) with the thrust vectors passing through the centroid of the CubeSat. For a nominal on-orbit mission, ${ }^{3}$ the center of mass $(\mathrm{CM})$ is designed such that it shall not vary by more than $1 \mathrm{~cm}$ from the centroid. As a result, the disturbance torques from the thruster firings would be kept at a minimum. Three reaction wheels are used to provide fine attitude control to the CubeSat. The simplicity of the design minimizes the number of control effectors and allows de-coupling of the translational and attitude loops.

\footnotetext{
* GNC Lead, Vehicle Analysis Branch

${ }^{\dagger}$ Graduate Student

${ }^{\ddagger}$ Graduate Student

$\S$ Associate Professor

ฯ Undergraduate Student
} 
The CubeSat control allocation algorithm (i.e. mixer) takes the acceleration (impulse) command at each control cycle $(3 \times 1$ vector $)$ and transforms it into RCS thruster on-times $(4 \times 1)$. Since there are more control effectors than degrees of freedom, the solution to the control allocation problem is overdetermined. ${ }^{4,5}$ The objective is to find the on-times that would minimize the error between the commanded impulse and the impulse delivered at each control cycle subject to on-time constraints. In this case, the thruster on-time is confined to be between 0.02 and 0.2 seconds. Equation 1 illustrates the error minimization problem: ${ }^{6}$ given the observability and control effectiveness matrices $C$ and $B$ respectively, find a vector $u$ such that

$$
J=\left\|C B u-a_{d}\right\|
$$

is minimized, subject to $u_{\min } \leq \mathrm{u} \leq u_{\max }$. Here $a_{d}$ is the desired 3-axis acceleration command, $u$ is the thruster on-times.

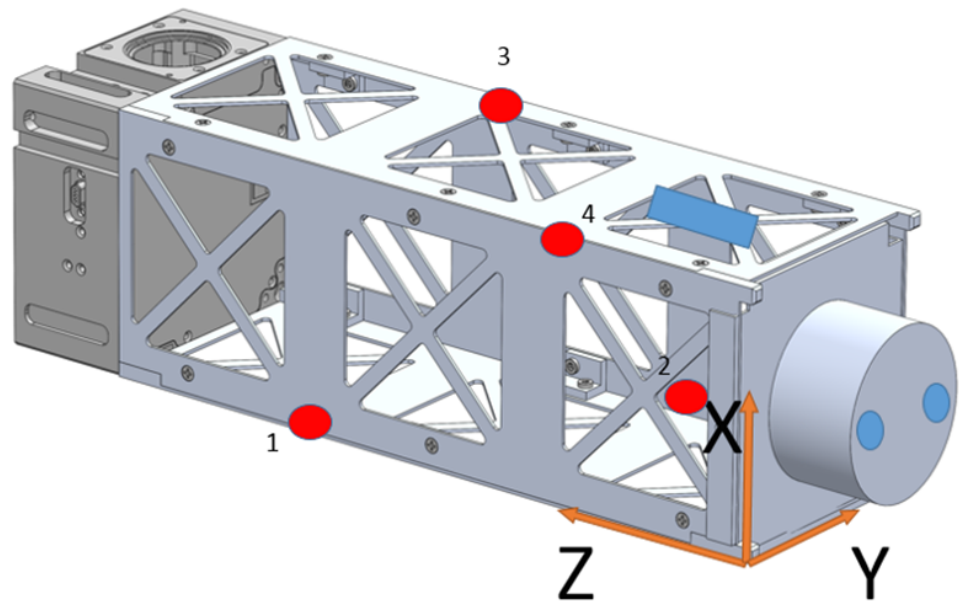

Figure 1. Thruster Layout

Compared to continuous control effectors (aerodynamic surfaces, thrust vector control, reaction wheels, etc.) reaction thrusters are more complicated because: 1$)$ they are inherently nonlinear (ON/OFF) with the impulse delivered proportional to the on-time, 2) they are uni-directional in most cases (can only produce one-sided force or torque). Generally for simplicity, ease of implementation, and compatibility with linear analysis, a linear control allocation scheme via pseudo inverse is preferred. Reference 7 offers an excellent explanation of the pseudo inverse control allocation scheme for a four thruster RCS configuration. At each control cycle, the pseudo inverse of the $B$ matrix (magnitude and direction of individual thrusters) maps the commanded impulse into thruster on-times. Since the thrusters are uni-directional, one of the resultant ontimes from the pseudo inverse solution will be negative. Negative duty cycles cannot be physically realizable since the thrusters cannot be activated for negative time (thrusters are not bi-directional). As a result, one of the rows in the pseudo inverse of the $B$ matrix is ignored to make the algorithm operational.

The RCS pseudo inverse algorithm has two major assumptions: 1) the impulse vs. on-time curve is a straight line with the slope being the idealized thrust, and 2) when two or more thrusters fire simultaneously, the resultant force is the vector sum of the forces that are provided when the thrusters are firing individually. With the current propulsion design, neither one of the above assumptions holds. The resultant impulse delivered is unique to the thruster firing combinations and it is nonlinear with thruster on-time. As a result, an iteration-free modified pseudo inverse (MPI) algorithm was developed specifically to address these two known deficiencies such that the error between the impulse command and impulse delivered is minimized. This algorithm is crucial during the docking phase of the mission since it requires high precision from the RCS. The remainder of this paper is organized as follows: Section II describes the performance of the propulsion system. Characterization test results are shown to illustrate the significant interactions between thruster firing combinations. Section III describes the MPI algorithm. Section IV compares the performance of the algorithm to the standard pseudo inverse and numerical optimization solutions. Sections V and VI provides further discussions and summarizes the findings. 


\section{Propulsion System Performance}

Successfully docking two Cubesats with small docking interfaces demands a highly precise RCS. The design of the thrusters came down to a tradeoff between minimizing the undesirable effects of the minimum impulse bit ${ }^{7}$ (MIB) by keeping thruster throat diameter small while still maintaining consistency in the performance characteristics. The definition of MIB used for this project is: 1) the impulse at which reliable measurement is possible, and 2) after the MIB, impulse vs. on-time behaves linearly. It was observed during testing that impulse vs. on-time became erratic once the throat diameter dropped below a certain threshold. For the final design, the thrusters have a target throat diameter of $0.8 \mathrm{~mm}$ with a theoretical thrust of 0.3 N. The delivered thrust can be described by Eq. 2, where the exit pressure, $P_{e}$, and the ambient pressure, $P_{o}$, should be the same if the gas is properly expanded. The mass flow rate, $\dot{m}$, shown in Eq. 3 and exit velocity, $v_{e}$, shown in Eq. 4 are both explicit functions of the nozzle geometry and state parameters.

$$
\begin{gathered}
F=\dot{m} v_{e}+\left[p_{e}-p_{o}\right] A_{e} \\
\dot{m}=\frac{p_{o} A^{\star}}{\sqrt{T_{o}}} \sqrt{\frac{\gamma}{R} \frac{2}{\gamma+1}^{\gamma+1 / \gamma-1}} \\
v_{e}=\sqrt{\frac{T R}{M} \frac{2 \gamma}{\gamma-1}\left[1-{\frac{p_{e}}{p_{o}}}^{\gamma-1 / \gamma}\right]}
\end{gathered}
$$

Here, $\gamma$ is the specific heat ratio, $R$ is the gas constant, $M$ is the molar mass, $T_{o}$ is the ambient gas temperature, and $A^{*}$ is the nozzle throat diameter.

In order to characterize the thruster performance, a 3-axis test stand shown in Fig. 2 was constructed. Details of the experimental setup and post processing involved can be found in Ref. 8. The test setup enables measurements of all three forces and moments in the form of strain via known linear relationships. Wheatstone bridge DAQ (Data Acquisition) measures the change in resistivity of the strain gauges from thruster firings.

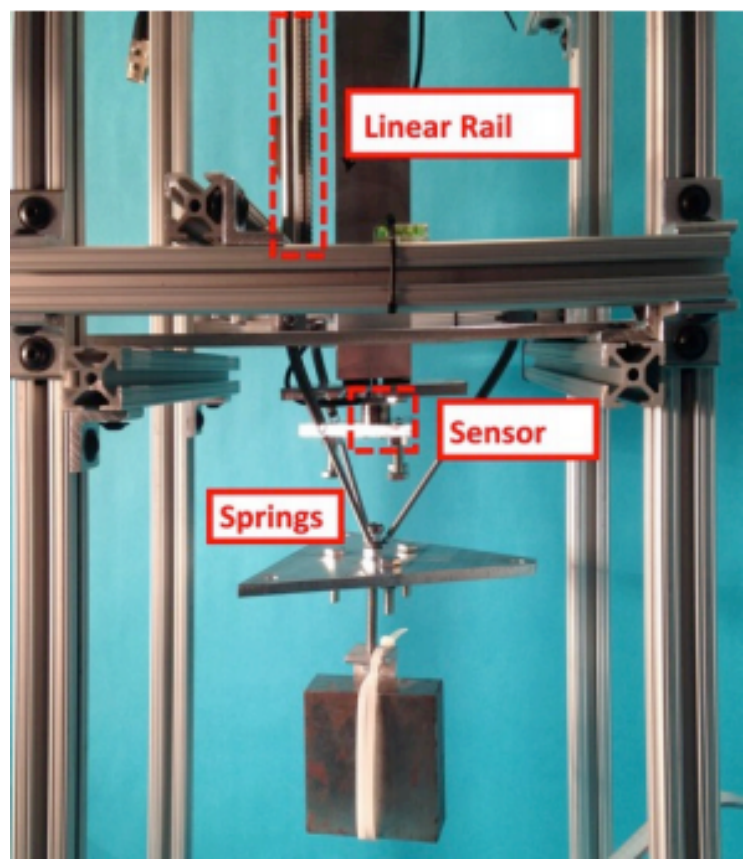

Figure 2. Propulsion Test Setup

For a given run during the characterization test, each thruster firing combination ( 1 thruster, 2 thruster, 3 thruster firings, 14 total) and on-times (0.02 to 0.2 seconds) were selected at random. The random approach was taken to mitigate systematic errors during testing whereby gradual reduction in the upstream tank 
pressure affects the impulse delivered. The resultant strain data was recorded and was then extensively postprocessed. For a given thruster firing combination, a nonlinear least-square approach was used to determine the best estimated thrust vector direction, $\hat{r}$, and impulse vs. on-time behavior. Multiple characterization tests were performed to ensure consistency from test to test.

Figure 3 shows impulse vs. thruster on-time results from three separate experimental tests (designated in green, red, and blue) for thrusters \#1 and \#2. The dots represent the test points and the solid line presents a least-squares fit through each data set. The average of the least-squares fits was used to come up with a mean model for simulation. For thruster \#1, the MIB is approximately $0.022 \mathrm{~N}$-s and the nominal thrust is around $0.3 \mathrm{~N}$. For thruster \#2, the MIB is slightly higher at around $0.028 \mathrm{~N}-\mathrm{s}$ and the nominal thrust is approximately $0.3 \mathrm{~N}$. Thrusters \#3 and \#4 (not shown here), exhibit similar characteristics.
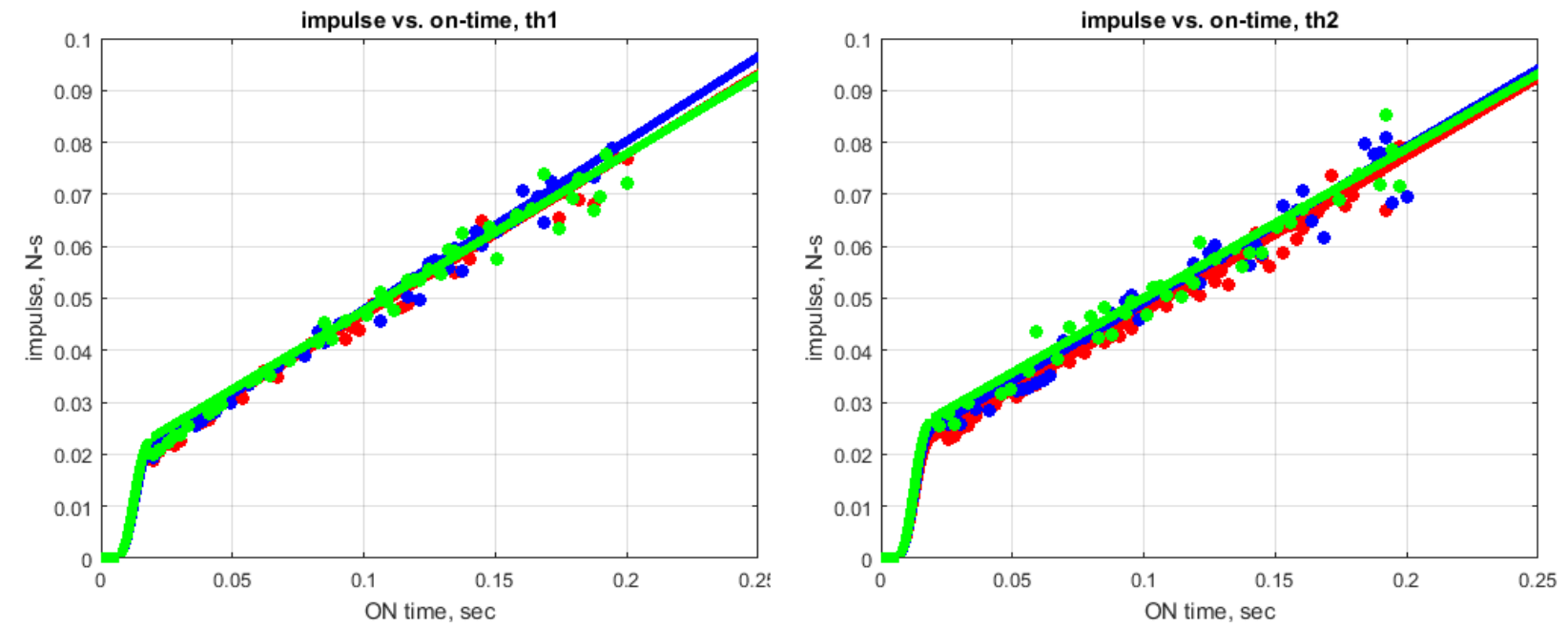

Figure 3. Impulse vs. Time, Thrusters \#1 and \#2

Table 1 compares the best estimated $\hat{r}$ with the ideal $\hat{r}$. Due to manufacturing imperfections (3D printing, installation, etc.), the best estimated thrust vector directions deviate by 15 to 20 deg from the theoretical directions. The best estimated $\hat{r}$ (for individual thruster firing combinations) are used to form the control effectiveness $(B)$ matrix.

Table 1. Thrust Vector Ideal vs. Best Estimated

\begin{tabular}{ccccccc}
\hline \hline Thruster & X ideal & Y ideal & Z ideal & X BE & Y BE & Z BE \\
\hline 1 & 0.4652 & 0.5443 & -0.6981 & 0.3578 & 0.7788 & -0.5152 \\
2 & 0.4652 & -0.5443 & 0.6981 & 0.4779 & -0.6446 & 0.5968 \\
3 & -0.4652 & -0.5443 & -0.6981 & -0.3734 & -0.7671 & -0.5216 \\
4 & -0.4652 & 0.5443 & 0.6981 & -0.3993 & 0.7044 & 0.5868 \\
\hline \hline
\end{tabular}

Figure 4 shows similar impulse vs. on-time plots for two thruster firing combinations $(\# 1,2$ and \#2,3). Once again, the dots represent the test points and the solid line presents a least-squares fit through each set (designated in green, red, and blue). Due to the regulator and tank design, it was observed that the net impulse delivered when two thrusters are firing is not a sum of the individual thruster firing results (same $\hat{r}$ but impulse magnitude is off by a scale factor depending on the firing combination). Figure 5 is an overlay plot of the experimental data (from a single test) for thrusters \#1,2 firing combination vs. the sum of thruster \#1 and \#2 individual firings. For all the multi-thruster firing combinations, the measured impulse underperform compared to the sum of individual firings. 

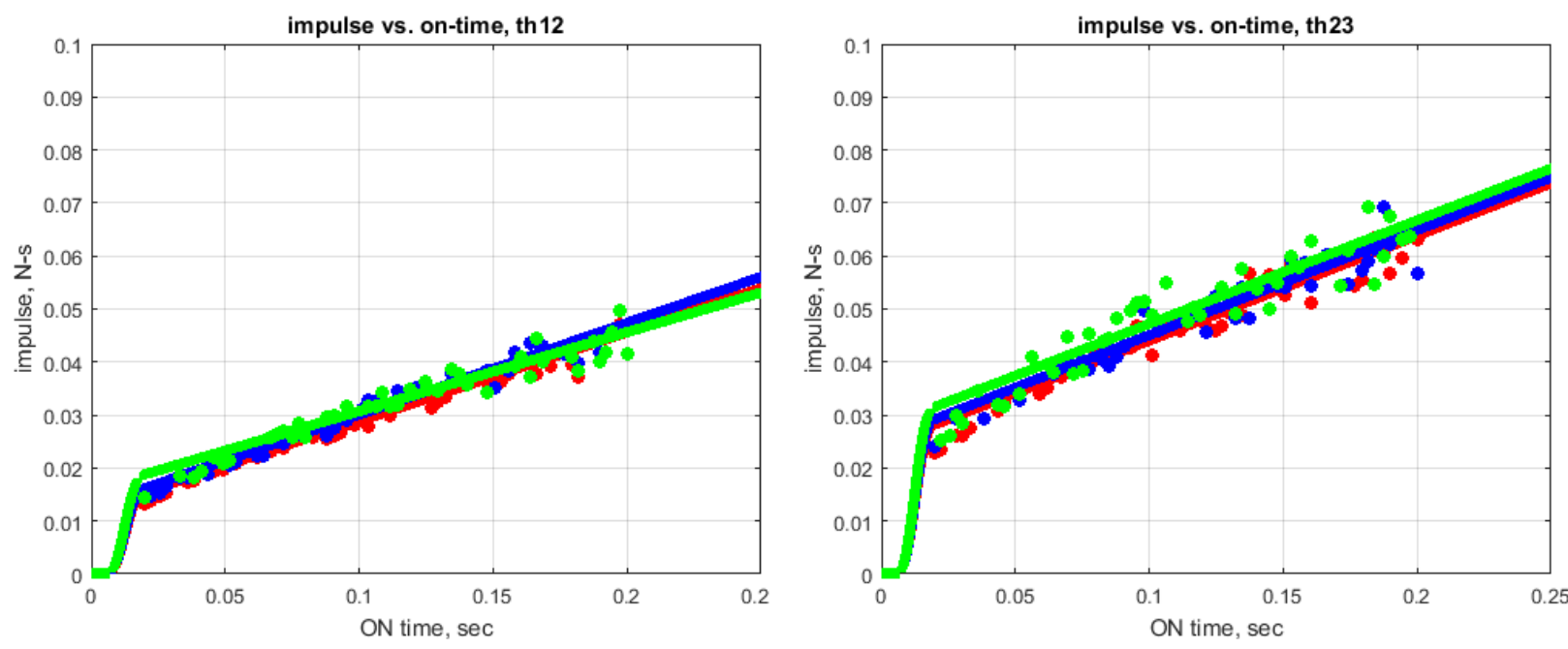

Figure 4. Impulse vs. Time, Thruster \#1,2 and \#2,3

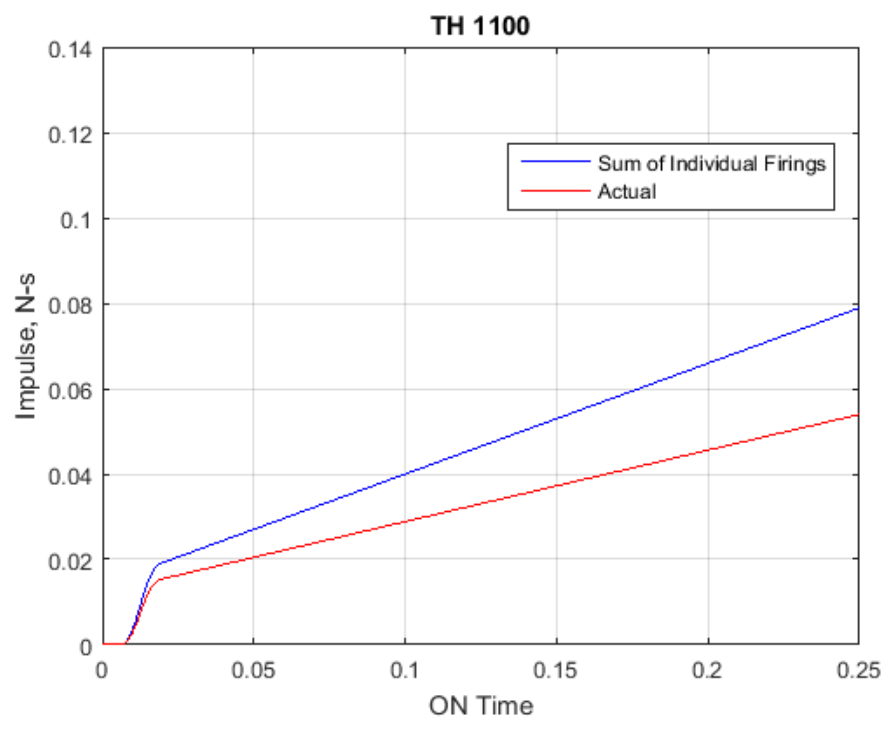

Figure 5. Impulse vs. Time, Thruster \#1,2 firing vs. sum of individual firings from Thruster \#1 and \#2

This phenomenon was apparent in the pre-regulator pressure readings, as a drop in pressure immediately after a firing is more significant when more than one thruster is firing. The OAAN propulsion team suspects the current regulator design has a varying mass flow rate for different thruster firing combinations. Reference ${ }^{9}$ describes similar observations for RCS systems. Figure 6 shows the internal layout of the current RCS design. 


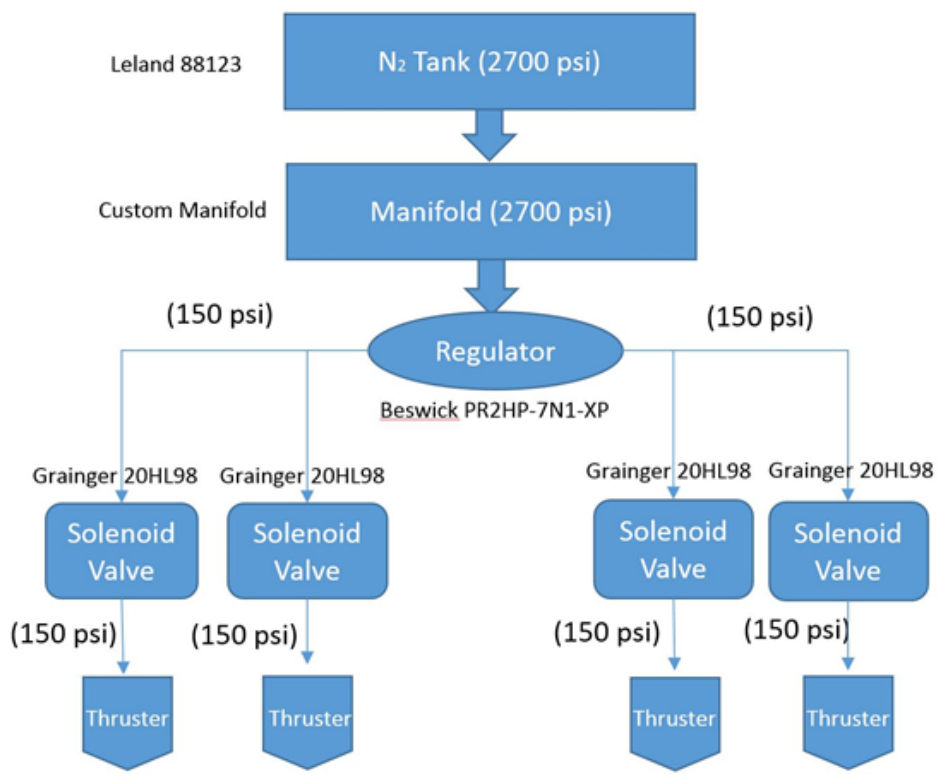

Figure 6. RCS Internal Layout

Figure 7 shows impulse vs. on-time plots for three thruster firing combinations (\#1,2,3 and \#2,3,4). The deviation in the impulse delivered compared to the sum of individual thruster firing is even more significant compare to the two thruster firing scenarios. This undesirable behavior creates additional complexity from a modeling and simulation perspective as a separate force vs. on-time model (similar to Figure 8) needs to be created for each thruster firing combination (14 total). Given the best estimated $\hat{r}$ and impulse vs. on-time data, a force (integral of force is impulse) vs. on-time model can be generated for each thruster firing combination. Figure 8 contains sample plots of the three-axis force vs. on-time models for thruster \#1 and \#1,2,3 combinations. The initial dead zone during the first 0.005 seconds is associated with opening of the thruster valve. The sharp transient in the following 0.015 seconds is a typical behavior exhibited by cold gas thrusters. After 0.02 seconds, the thrust delivered is constant with on-time.
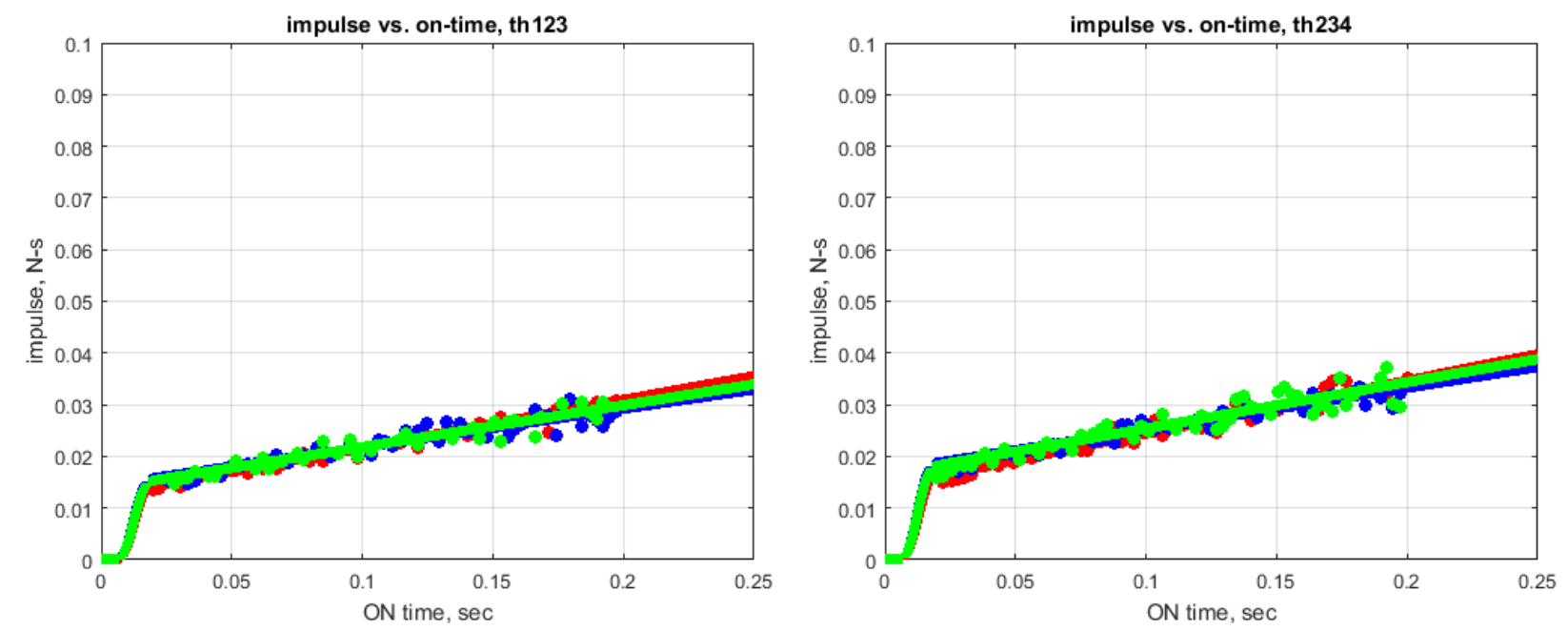

Figure 7. Impulse vs. Time, Thrusters \#1,2,3 and Thrusters \#2,3,4 

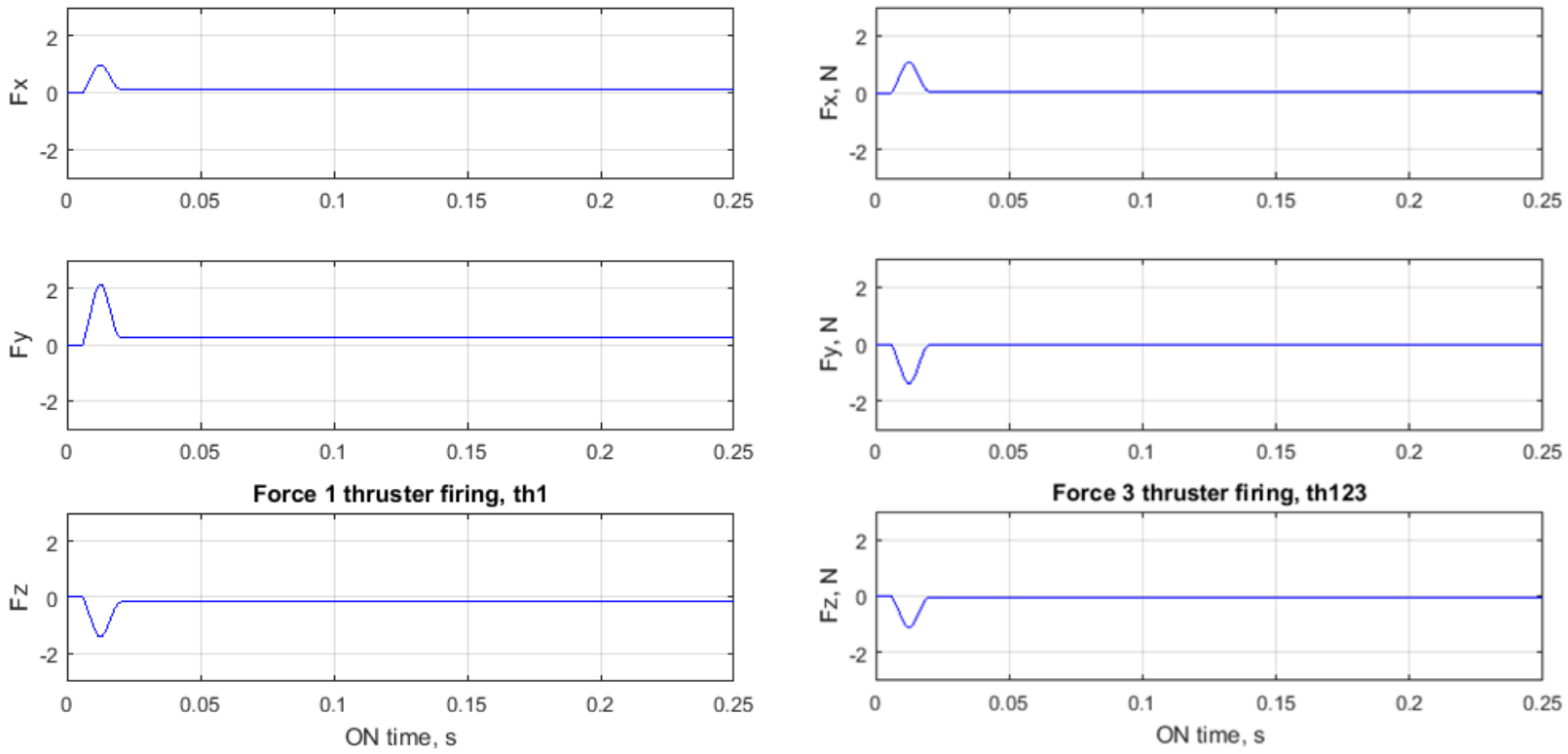

Figure 8. Force vs. Time model, Thruster \#1 and Thruster \#1,2,3

An excellent way to visualize the controllability space of the current RCS design is to examine the theoretical attainable impulse set, $\Phi .{ }^{4,5}$ The theoretical unattainable impulse set, $\Theta$, is a sub-region of $\Phi$ which the RCS design cannot access due to thruster MIB. Figure 9 illustrates $\Phi$ and $\Theta$ for the ideal RCS design (no thrust vector misalignments and the resultant force of two or more thrusters firing is the sum of the individual thrusters). These volumes were subsequently computed using the convex hull algorithm in MATLAB. ${ }^{10}$ For the ideal RCS design and the thruster on-time constraints, the $\Phi$ and $\Theta$ are $0.0016(N s)^{3}$ and $5.98 \mathrm{e}-5(N s)^{3}$ respectively.

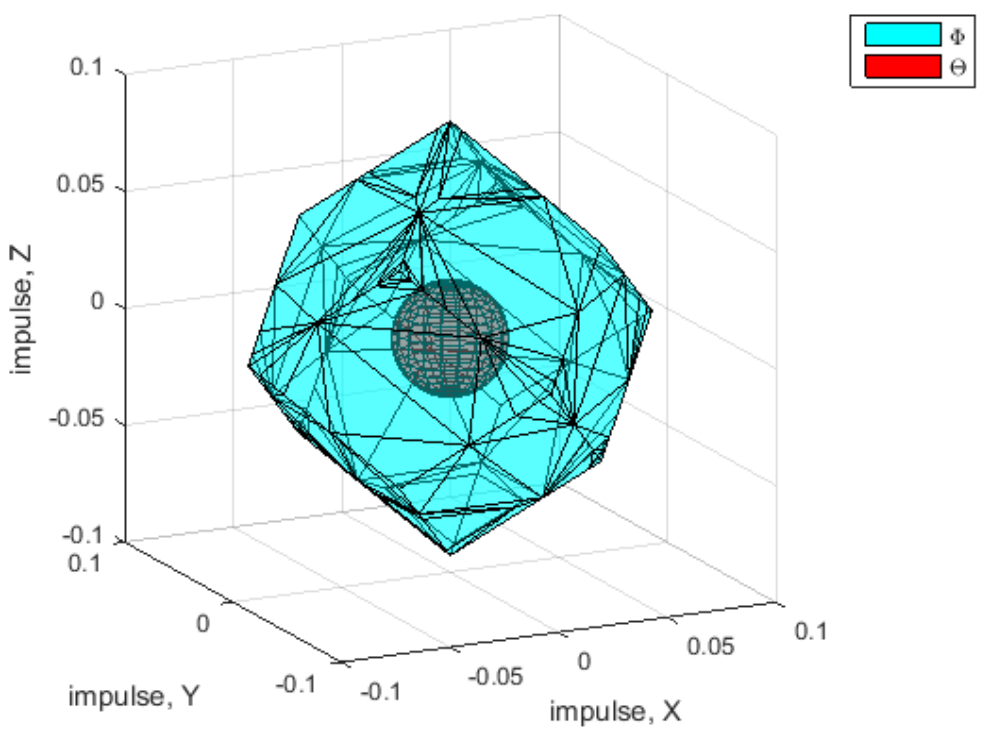

Figure 9. $\Phi$ and $\Theta$ for Ideal RCS Design

Figure 10 shows $\Phi$ and $\Theta$ for the actual RCS design. Due to the described deficiencies in the design, the symmetrical topographic nature of $\Phi$ and $\Theta$ is lost. $\Phi$ and $\Theta$ volumes are $0.00049(N s)^{3}$ and 5.06e-6 $(N s)^{3}$ respectively. Figure 11 overlays $\Phi$ of the actual RCS design with the idealized. The control allocation 
efficiency, ${ }^{5} \eta$, in this instance is defined as the volume ratio of $\Phi$ for the actual design to the ideal design. The actual system can only achieve approximately $31 \%$ of the ideal $\Phi$ which highlights the inefficiency of the current design.

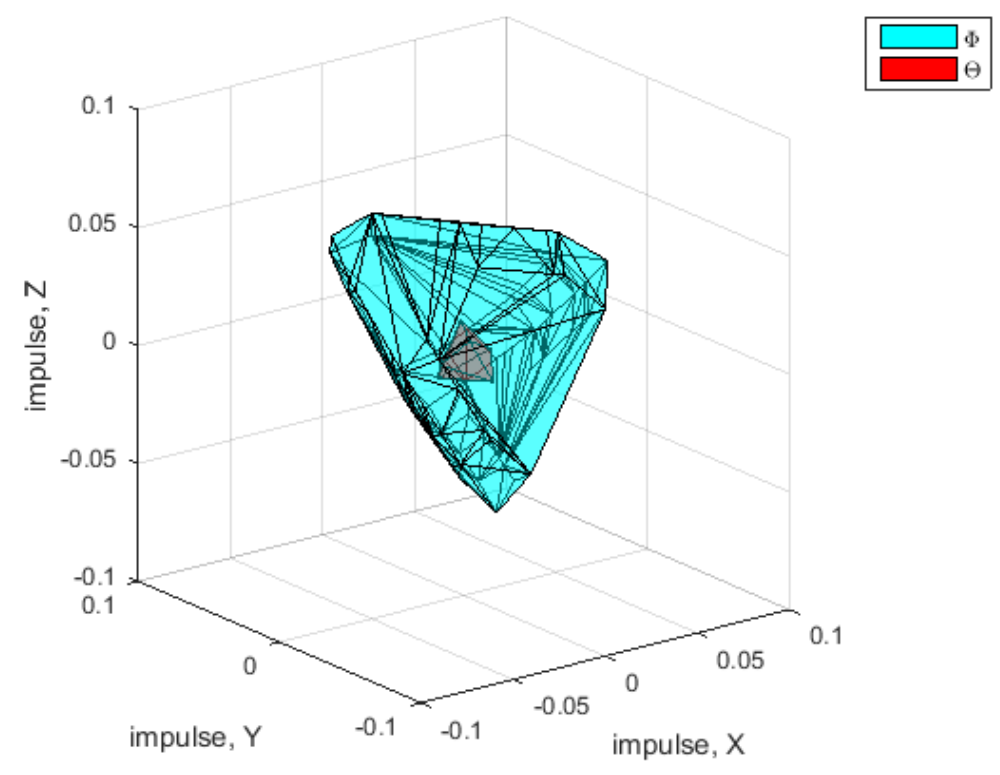

Figure 10. $\Phi$ and $\Theta$ for Actual RCS Design

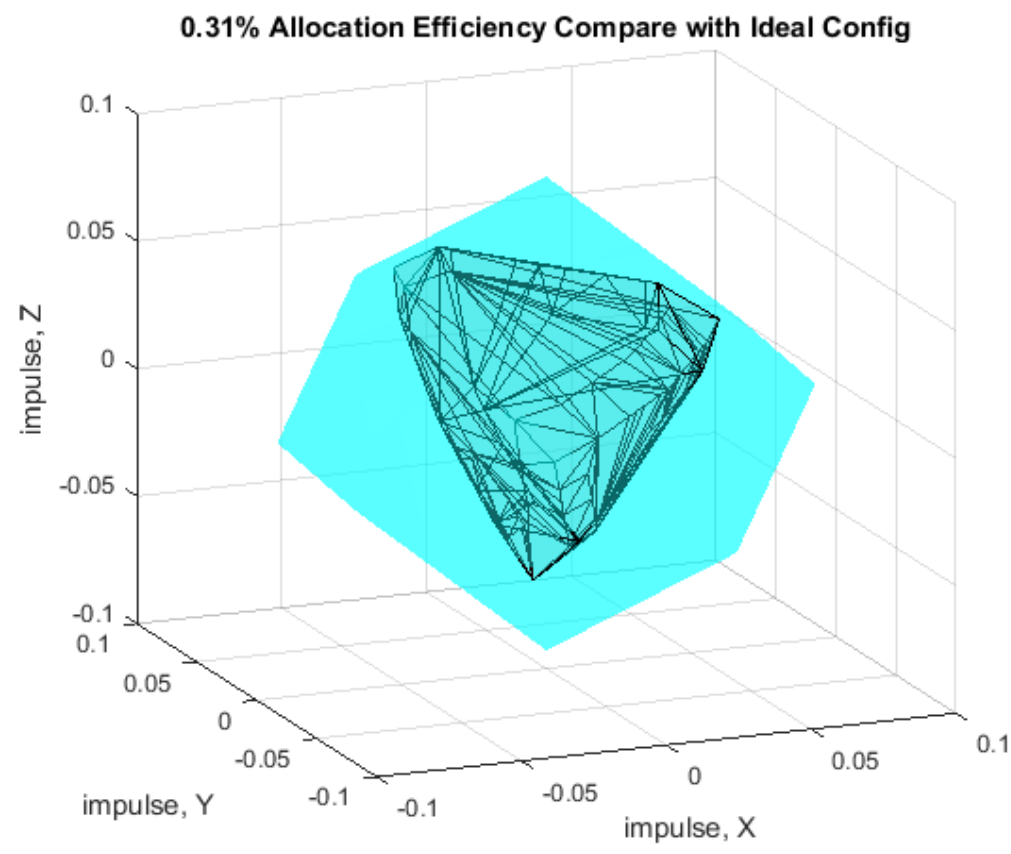

Figure 11. $\Phi$ Ideal vs. Actual RCS Designs 


\section{Modified Pseudo Inverse Algorithm}

Generally, errors introduced in the control allocation solution are mitigated by the feedback control law. However, this approach is rather "reactive", an undesirable characteristic considering the precision required to dock two CubeSats. Furthermore, if the GNC is operating in a more open loop fashion (i.e. applying a $\Delta \mathrm{V}$ to a spacecraft during a rendezvous mission), the resultant control allocation errors would result in large deviations in the spacecraft trajectory. Due to deficiencies with the current RCS design, the traditional pseudo inverse solution would yield large errors between the commanded and delivered impulse. As previously stated, the pseudo inverse algorithm assumes: 1) impulse vs. on-time curve is a straight line with the slope being the idealized thrust, 2) the resultant force from two or more thrusters firing is the sum of the individual contributions. Test results show neither one of these assumptions hold for the current design. Figure 12 is a plot of the actual and ideal impulse vs. on-time for thruster \#1. The actual impulse vs. on-time curve is nonlinear. Tests show if the on-time is below the opening and closing time of the solenoids $(0.005 \mathrm{sec})$, the impulse delivered is zero. The time in between the min on-time of the solenoids and when steady thrust is reach is considered the transient portion $(0.005$ to $0.02 \mathrm{sec})$.

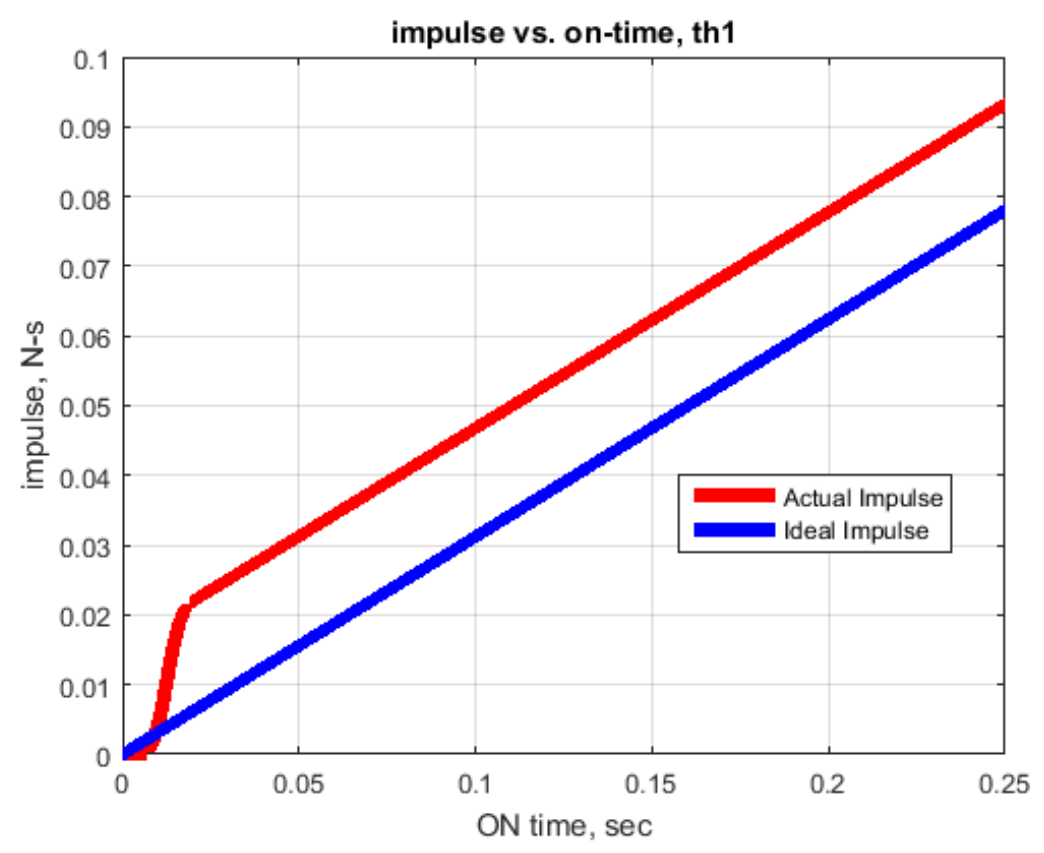

Figure 12. Actual and Ideal Impulse vs. on-time

Usually, the control allocator requires a linear approximation of the control effector input. Doman ${ }^{11}$ addressed the problem of having nonlinearities in the control effectiveness matrix by providing an additional intercept term that corrects for the errors introduced by the assumption of linearity. The approach adjusts the $B$ matrix depending on the current operating condition. Few works in the literature address control effector interactions in the control allocation problem. In most cases, the interaction effects are small and hence ignored in the control allocation schemes. Oppenheimer ${ }^{12}$ showed an iterative linear programming approach to include control effector interaction in the aircraft control allocation problem and applied the method to a multi-gimbal thruster rocket configuration. To the authors' knowledge, there exists no literature for RCS jets in which the interaction effects are not attributed to the mixing of the plumes but rather to the design of the upstream regulator. The proposed scheme is an iteration-free modified version of the pseudo inverse solution that takes into consideration test data for all 14 thruster firing combinations. Here are two key assumptions to the proposed algorithm:

1. The RCS design could achieve an arbitrary impulse vector in any direction and magnitude subject to on-time constraints by firing the thrusters in three sequential stages. For instance, three thrusters firing $(\# 1,2,4)$, followed by two $(\# 2,4)$, then by one $(\# 1)$. The number of thrusters firing has to decrease 
monotonically and the on-time for the current stage must be greater than the one prior. Given these constraints, there exist 24 unique combinations in which the thruster firing sequence could take place. This is referred to as the truth table and shown in Table 2. Here, columns 2-4 represent the unique thruster firing combinations for stage three, columns 5-6 for stage two, and column 6 for stage one.

2. The force vs. on-time for all 14 firing combinations ( 3 thrusters, 2 thrusters, 1 thruster firing) are repeatable and can be superimposed linearly. For instance, if the on-time commands for thrusters \# 1, 2, 4 are: $0.04,0.06,0.08$ seconds respectively, one would first evaluate the force vs. on-time lookup table for thruster combination \#1, 2, 4 from 0 to 0.04 seconds. Then, one would evaluate the force vs. on-time lookup table for thruster combination \#2, 4 from 0.04 to 0.06 seconds. Finally, one would evaluate the force vs. on-time lookup table for thruster combination \#4 from 0.06 to 0.08 seconds. The total force is the sum of the forces from the three stages.

Table 2. Modified Psuedo Inverse Algorithm: Truth Table

\begin{tabular}{lllllll}
\hline \hline 1, & 2 & 3 & 4 & 3 & 4 & 4 \\
2, & 2 & 3 & 4 & 3 & 4 & 3 \\
3, & 2 & 3 & 4 & 2 & 4 & 4 \\
4, & 2 & 3 & 4 & 2 & 4 & 2 \\
5, & 2 & 3 & 4 & 2 & 3 & 3 \\
6, & 2 & 3 & 4 & 2 & 3 & 2 \\
7, & 1 & 3 & 4 & 3 & 4 & 4 \\
8, & 1 & 3 & 4 & 3 & 4 & 3 \\
9, & 1 & 3 & 4 & 1 & 4 & 4 \\
10, & 1 & 3 & 4 & 1 & 4 & 1 \\
11, & 1 & 3 & 4 & 1 & 3 & 3 \\
12, & 1 & 3 & 4 & 1 & 3 & 1 \\
13, & 1 & 2 & 4 & 2 & 4 & 4 \\
14, & 1 & 2 & 4 & 2 & 4 & 2 \\
15, & 1 & 2 & 4 & 1 & 4 & 4 \\
16, & 1 & 2 & 4 & 1 & 4 & 1 \\
17, & 1 & 2 & 4 & 1 & 2 & 2 \\
18, & 1 & 2 & 4 & 1 & 2 & 1 \\
19, & 1 & 2 & 3 & 2 & 3 & 3 \\
20, & 1 & 2 & 3 & 2 & 3 & 2 \\
21, & 1 & 2 & 3 & 1 & 3 & 3 \\
22, & 1 & 2 & 3 & 1 & 3 & 1 \\
23, & 1 & 2 & 3 & 1 & 2 & 2 \\
24, & 1 & 2 & 3 & 1 & 2 & 1 \\
\hline \hline & & & & & & \\
\hline & & & &
\end{tabular}

Figure 13 is a schematic of the algorithm and works as follows:

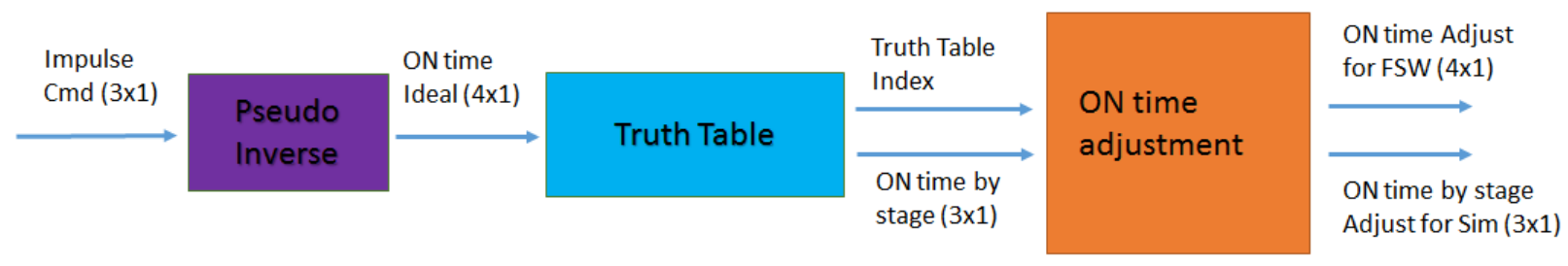

Figure 13. Control Allocation Schematic: Modified Pseudo Inverse 
1. From the impulse command $(3 \times 1)$, determine the idealized thruster on-times $(4 \times 1)$ based on standard pseudo inverse solution.

2. From the idealized on-times $(4 \times 1)$ sort the on-times by stages $(3 \times 1)$ and find the corresponding index in the truth table.

3. Adjust the idealized stage on-times $(3 \times 1)$ by the experimental impulse data $(\# 3,2,1$ thruster firing combinations), ie. Fig 12. Apply stage on-time limits: if the adjusted stage on-time is 0.02 sec or less set the on-time to 0 , if the adjusted stage on-time is greater than 0.2 set the on-time to $0.2 \mathrm{sec}$.

4. Convert the stage on-times $(3 \times 1)$ to thruster on-times $(4 \times 1)$ for flight software $(F S W)$. The stage on-times are used to estimate the impulse delivered per control cycle.

The proposed algorithm does not involve iterations and it is simple for flight software implementation. The idealized and experimental impulse vs. on-time for each thruster firing combination (14 total) are stored as lookup tables. A simple search algorithm is required to find the corresponding row in the truth table given the stage on-times.

\section{Numerical Results}

The main objective of the allocation problem ${ }^{4}$ is to find a control vector, $u$, that results in the best approximation of the impulse or moment command vector, $a_{d}$, in the given direction. Reference 5 refers to this characteristic as co-linearity, an important characteristic for any allocator. A sensitivity study was performed to compare the accuracy of the three allocation schemes: 1) Pseudo Inverse, 2) Modified Pseudo Inverse, 3) Numerical optimization using MATLAB's optimization toolbox (not feasible for real-time implementation). In this study, a table of impulse commands $(3 \times 1$ vector) ranging from -0.06 to 0.06 Ns in the $\mathrm{Y}$ and $\mathrm{Z}$ channels ( 0 command in the $\mathrm{X}$ channel) was feed individually into each allocation schemes. The error between the actual impulse delivered with the command is presented as a ratio between the magnitude of the two vectors (in $\mathrm{dB}$ ) and a single rotation angle (degrees).

Figure 14 shows the error between the impulse delivered with the command for the pseudo inverse approach. The results are color-coded by the magnitudes of the commanded impulse (red circles represent commands with $l_{2}$ norms $<0.03 \mathrm{Ns}$ and blue circles for commands $>0.03 \mathrm{Ns}$. Even without performing this sensitivity study, it is apparent from the nonlinear impulse vs. on-time test data shown in Fig. 12 that the pseudo inverse solution will yield a delivered impulse that is greater than the commanded. The sensitivity analysis results validate the claim as majority of the red circles show the magnitude of the impulse delivered to well exceed the commanded. The direction errors lie somewhere between 0 and 30 degrees. When the CubeSats are within close proximity with their docking interfaces pointed at each other, the controller issues fine commands to the chaser such that it slowly enters the "basin of attraction" of the magnetic docking system with minimal kinetic energy. ${ }^{1}$ The overshoot characteristic of the pseudo inverse scheme is undesirable by subjecting the chaser to a greater impulse than anticipated and causing it to exit the "basin of attraction" which increases the likelihood of in a failed docking scenario. 


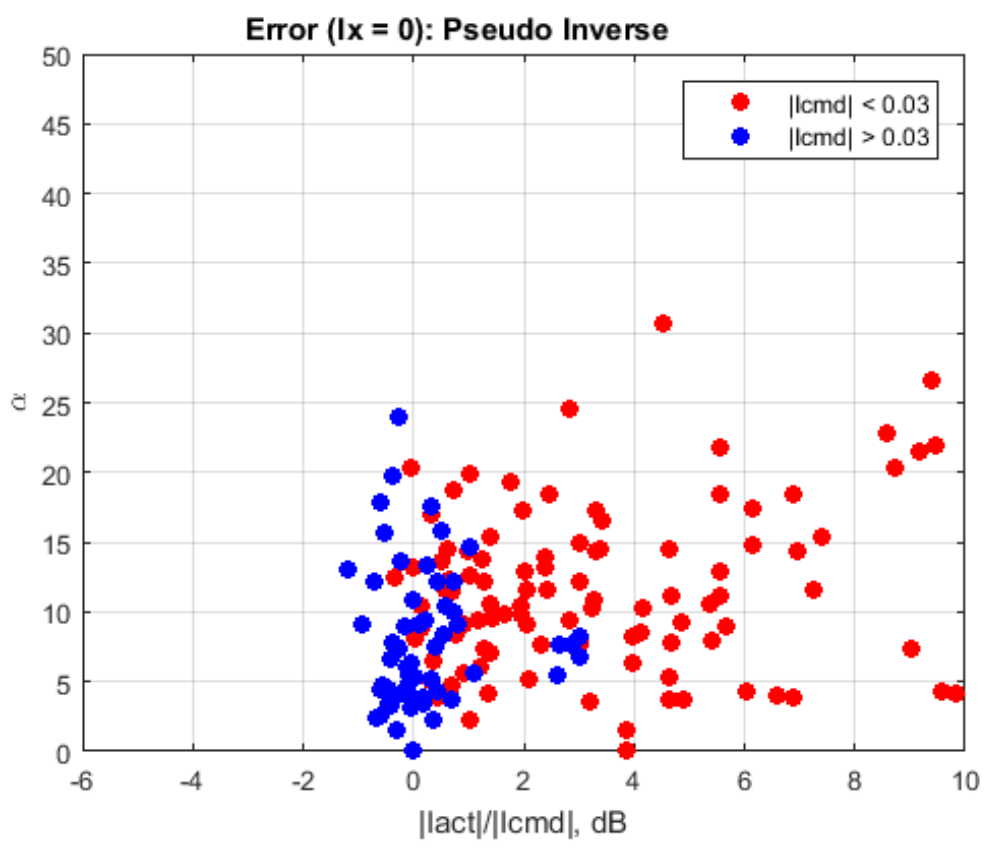

Figure 14. Allocation Error: Pseudo Inverse

The MPI solution adjusts the on-time solutions from pseudo inverse based on experimental data. Due to the unique characteristics in the performance of the propulsion subsystem, the on-times from MPI is always smaller compare to pseudo inverse. Figure 15 shows the error between the impulse delivered and the impulse commanded for the MPI algorithm. It is apparent that the MPI allocator yields solutions that consistently underperform compare to the commanded. This undershoot behavior is more desirable for the docking phase of the mission. In comparison with standard pseudo inverse, with MPI there exists a greater region in the commanded impulse space for which the algorithm cannot access due to the hard minimal on-time constraint. A key GN\&C metric for the docking phase is to have the chaser CubeSat approach the "basin of attraction" of the magnetic docking subsystem with minimal kinetic energy. 


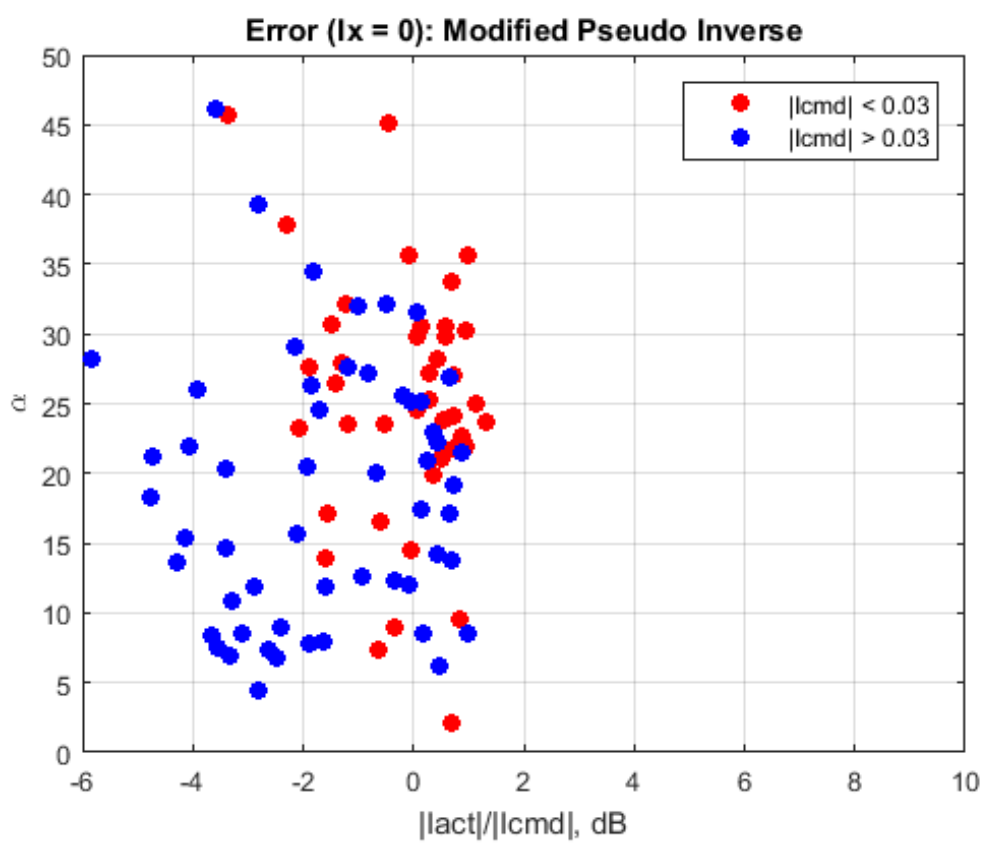

Figure 15. Allocation Error: Modified Pseudo Inverse

In the third study, the control allocation problem was posed as an numerical optimization problem with an objective function and constraints solved using MATLAB's fmincon function. ${ }^{10}$ fmincon is a gradient descent type of algorithm which is not feasible for real-time implementation. Figures 16 and 17 show the errors between the delivered and commanded impulses. Without constraints on the min and max thruster on-times, numerical optimization can achieve the commanded impulse with near perfection with larger errors for commands near the edges of the impulse command space. Figure 17 shows errors between the delivered and commanded impulses with constraints on the thruster on-times considered. For most commands, the numerical optimization solution is superior to both pseudo inverse and MPI; however for certain commands in the impulse command space it exhibits a similar overshoot type of behavior as with the pseudo inverse. 


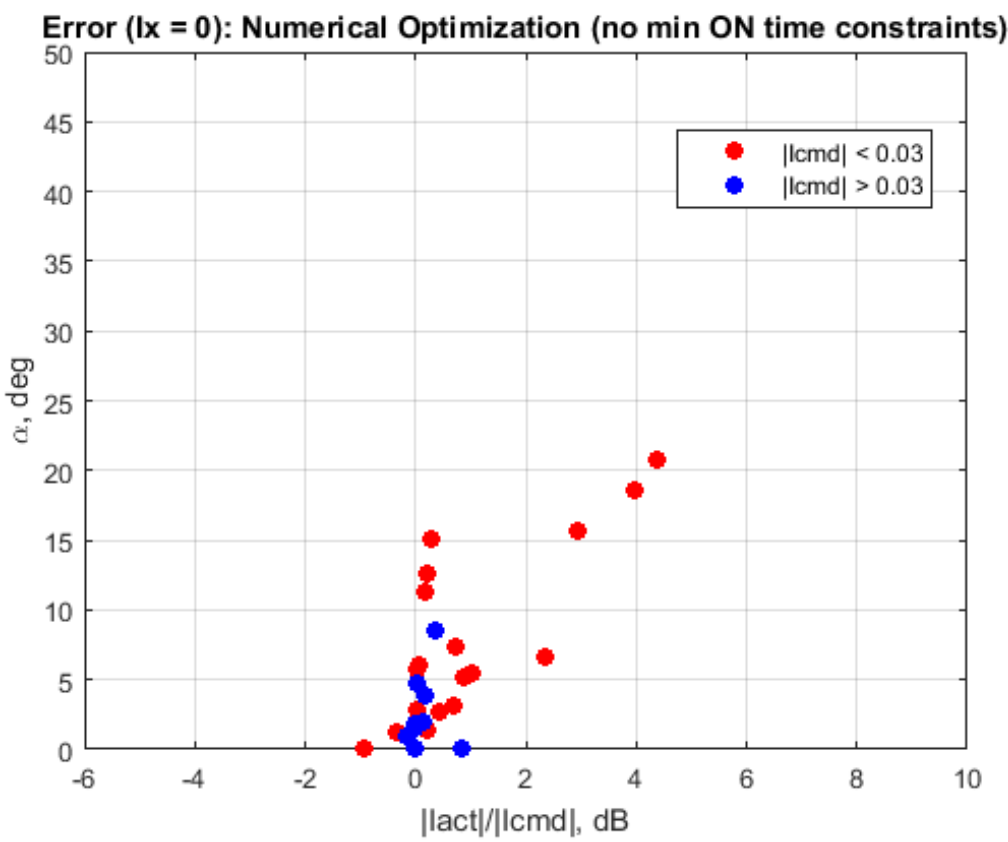

Figure 16. Allocation Error: Numerical Optimization with no on-time Constraints

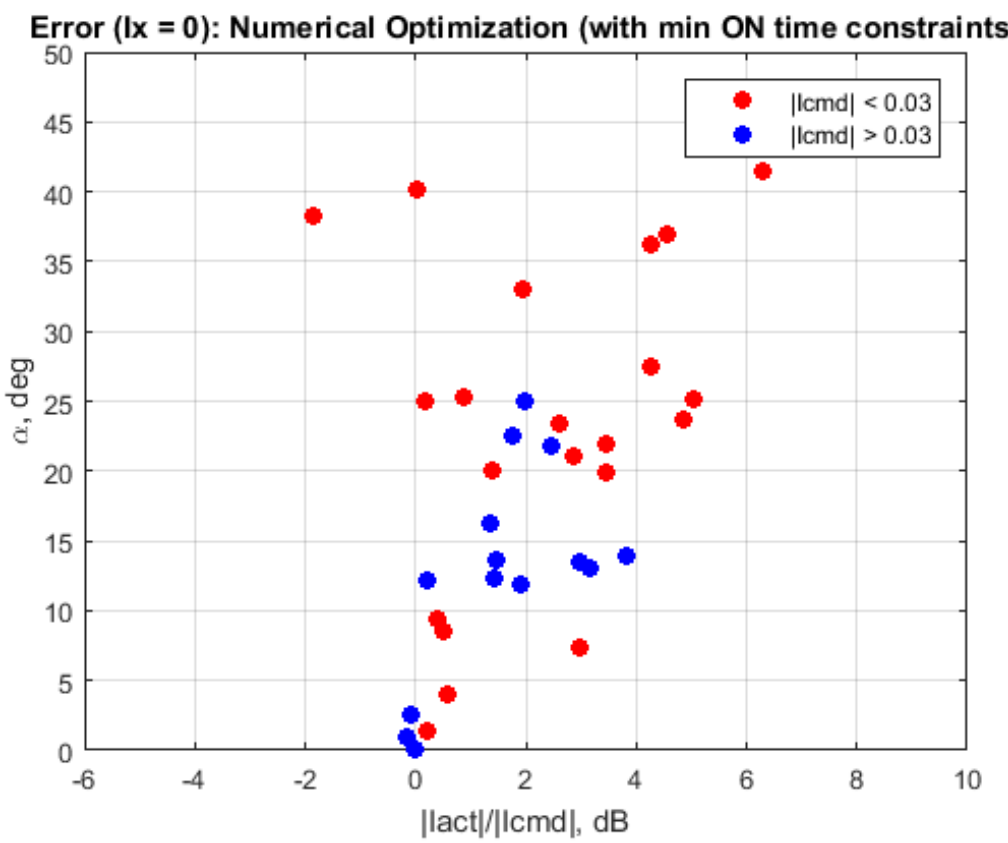

Figure 17. Allocation Error: Numerical Optimization with on-time Constraints

It is apparent from the sensitivity study described above that given the uniqueness of the RCS performance, the MPI algorithm exhibits an undershoot behavior that is more desirable for the docking phase of the mission compared to the standard pseudo inverse. Another challenging problem in the evaluation of control allocation schemes ${ }^{4,5}$ is determining the shape of the attainable impulse or moment set that is achievable using a particular allocation strategy and comparing it with the theoretical attainable maximum $(\Phi)$ subject to the constraints. The particular attainable impulse set, $\Phi^{*}$, was evaluated using the MPI algorithm. Figure 18 shows an overlay of $\Phi^{*}$ with $\Phi$. $\Phi^{*}$ was generated using a similar bisection brute force 
approach discussed in Orr. ${ }^{5}$ It is well known that with a generalized inverse, only a subset of the admissible controls maps to the boundary of the theoretical attainable set. The control allocation efficiency, $\eta$, here is defined as the ratio between the particular and the theoretical sets shown in Eq. 5. The MPI solution can achieve approximately $81 \%$ of the theoretical attainment impulse set.

$$
\eta=\frac{\Phi^{*}}{\Phi}
$$

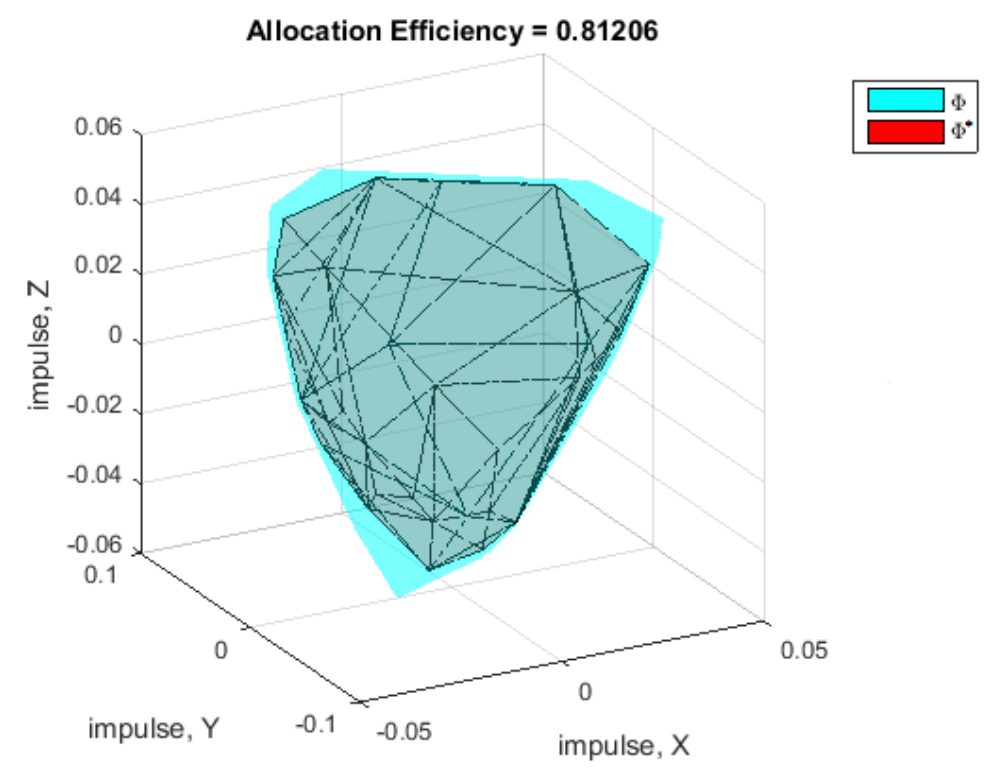

Figure 18. $\Phi^{*}$ vs. $\Phi$ Actual RCS Design

\section{Discussion}

Unlike aircraft or launch vehicle control allocation problems where the primary objective is to maximize the $\Phi$ and $\Phi^{*}$, the spacecraft rendezvous and docking problem is most concerned with minimizing the effects of $\Theta$ and $\Theta^{*}$ that arise due to thruster MIB. As stated in Section II, the MIB largely depends on the physical parameters of the thrusters and was considered in the RCS design as a tradeoff between minimizing the throat diameter and data repeatability. With the MPI allocation scheme, there exists a small region in the impulse space that the algorithm cannot access as shown in Fig 10. This is equivalent to having a dead zone in the open loop transfer function. A MIB mitigation algorithm ${ }^{2}$ was developed to compensate for this deficiency and was shown to be superior to the performance of an integral gain for this application. Figure 19 shows the block diagram illustrating this concept. 


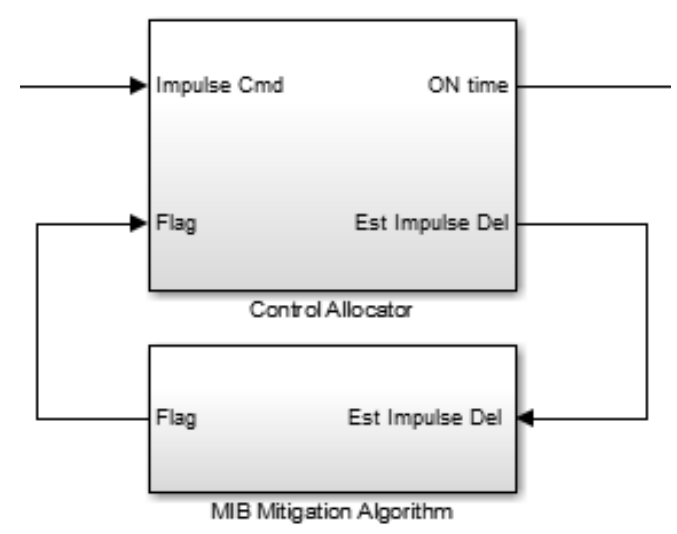

Figure 19. MIB Mitigation Algorithm

The basic concept of the MIB mitigation algorithm is as follows: estimate using the RCS model whether an impulse was applied to the vehicle during the current control cycle based on the commanded on-times. If the estimated impulse delivered is non-zero, proceed with the next control update (flag $=1$ ). Otherwise, (flag $=0$ ), start accumulation of the impulse command as follows: $I_{c m d}=I_{c m d, k}+I_{c m d, k-1}$. Here $k$ indicates the index associated with the current control cycle. This process continues until the impulse command is large enough to generate on-time commands that are greater than the minimum on-time constraints. This additional feature appears to be compatible with the undershoot nature associated with the MPI algorithm and increases the likelihood of a successful docking.

\section{Conclusion}

Design and manufacture of RCS for small spacecrafts is challenging due the miniaturized scale of all the hardware components. The paper highlights the unique deficiencies with the current four thruster layout design for the OAAN CubeSats. The modified pseudo inverse (MPI) algorithm was developed to address the problem of significant interactions between RCS jet firings. This problem is further complicated in that the interaction effect is unique for each thruster firing combination (14 total). The MPI algorithm is more desirable for the final docking phase of the mission compare to the standard pseudo inverse due to its undershoot nature and increases the likelihood of a successful docking. The algorithm is generic such that it could be applied to other configurations in which there are significant and unique interactions between control effectors. It is iteration free and fast enough for real-time implementation.

\section{Acknowledgement}

The authors would like to thank the NASA Science Technology Mission Directorate (STMD), Early Career Initiative (ECI), and Space Technology Exploration Directorate (STED) for funding to pursue this concept. The authors would also like to extend their gratitude to John Martin, RJ Bodkins, Carey Buttrill, Dr. Jeb Orr, Dr. John Davidson, Dr. Bandu Pamadi for their insights, and other members of the OAAN propulsion team at Cornell University for their significant contributions. 


\section{References}

${ }^{1}$ Pei J, Murchison, L. and et, "Autonomous Rendezvous and Docking of Two 3U Cubesats Using a Novel Permanent Magnet Docking Mechanism," No. 2016-1465, AIAA SciTech, 2016.

${ }^{2}$ Pei J, Murchison, L. and et, "Ground Demonstration on the Autnomous Docking of Two 3U Cubesats Using a Novel Permanent-Magnet Docking Mechanism," No. 2017-0849, AIAA SciTech, 2017.

${ }^{3}$ Pei J, Walsh, M. and et, "Preliminary GN\&C Design for the On-Orbit Autonomous Assembly of Nano-Satellite Demonstration Mission," AAS Conference Steveson WA, 2017.

${ }^{4}$ Durham, W., "Constrained Control Allocation: Three-Moment Problem," Journal of Guidance, Control, and Dynamics, Vol. 17, No. 2, Mar-Apr 1994.

${ }^{5}$ Orr, J. and Slegers, N., "High Efficiency Thrust Vector Control Allocation," Journal of Guidance, Control, and Dynamics, Vol. 37, No. 2, Mar-Apr 2014.

${ }^{6}$ Bodson, M., "Evaluation of Optimization Methods for Control Allocation," No. 2001-4223, AIAA, 2001.

${ }^{7}$ Sidi, M., Spacecraft Dynamics and Control, Cambridge University Press, 5th ed., 2006.

${ }^{8}$ Elandt R, Aslan, S. and Peck, M., "Six Axis thruster Characterization Technique for Small Satellites," TBD, 2017.

${ }^{9}$ Doman D, Gamble, B. and Ngo, A., "Control Allocation of Reaction Control Jets and Aerodynamic Surfaces for Entry Vehicles," No. 2007-6778, AIAA, 2007.

${ }^{10}$ Mathworks, MATLAB Optimization Toolbox, 2015.

${ }^{11}$ D.Doman and Oppenheimer, M., "Improving Control Allocation Accuracy for Nonlinear Aircraft Dynamics," No. 20024667, AIAA, 2002.

${ }^{12}$ Oppenheimer, M. and Doman, D., "A Method for Including Control Effector Interaction in the Control Allocation," No. 2007-6418, AIAA, 2007. 